\title{
Cancer Comprehensive Analysis in Gastric Carcinoma: Benefits and New Perspectives
}

\author{
Vasiliki Pisanidou ${ }^{a}$ Panagiotis Apostolou $^{b} \quad$ Georgios Beis $^{b}$ \\ Eleana Hatzidaki ${ }^{\text {b }}$ Ioannis Papasotiriou ${ }^{c}$ \\ a General Hospital of Ptolemaida "Mpodosakeio," Ptolemaida, Greece; 'besearch Genetic \\ Cancer Centre S.A., Florina, Greece; 'Research Genetic Cancer Centre International GmbH, \\ Zug, Switzerland
}

\section{Keywords}

Gastrointestinal cancer - Array comparative genomic hybridization · Next-generation sequencing $\cdot$ Microarrays

\begin{abstract}
Gastric cancer is one of the most common and deadly cancers worldwide. Screening tests as well as tools for prediction of treatment outcomes and prognosis have been developed, but they have many limitations. The integration of liquid biopsy provided new aspects in screening and diagnosis of gastric cancer. In the present study, we used different techniques, studying the genetic and epigenetic profile of circulating tumor cells. We aimed to acquire all the available information, compare it with already existing studies, and evaluate the benefit of this approach. A blood sample was isolated from 2 gastric cancer patients at stages III-IV, followed by the isolation of CTCS. The circulating tumor cells were used for array comparative genomic hybridization, next-generation sequencing, and whole gene expression microarrays. Different variants were detected, while the microsatellite instability status was stable in both cases. The tumor mutational burden was low to medium. Gene expression assays revealed that $>100$ genes were overexpressed compared to noncancer samples. Amplifications of $X$ chromosome were also observed in both cases, by using array comparative genomic hybridization. Although there are several techniques for cancer screening, prediction of therapy outcomes, and prognosis, the application of a complete comprehensive cancer panel, combining the study of variants, fusions, chromosomal abnormalities, and gene expression, is more appropriate. Information provided by the above techniques might contribute in designing more efficient
\end{abstract}

\section{Karger ${ }^{\prime \prime}$}


treatment protocols and screening tools. Despite the limitation of samples, the data are encouraging, and further study is needed so that they can be used at clinical level.

(C) 2021 The Author(s).

Published by S. Karger AG, Basel

\section{Introduction}

Gastric cancer, also known as stomach cancer, is one of the most common types of neoplasms worldwide. It is the third leading cause of cancer in the world, and over 1 million cases are diagnosed every year around the world [1]. This specific type of cancer is more prevalent in males, with approximately 2 times higher likelihood to affect males than females [2]. Five-year survival is highly associated with the stage of the disease, with patients at stage I having approximately $90 \%$ survival rate, while cases at stage III have only $18 \%$. In addition, in Europe, the 5-year survival rate is lower than in the USA [3]. Different risk factors have been associated with gastric cancer, such as chronic gastritis, inflammation, gastroesophageal reflux disease, or Helicobacter pylori-caused gastritis [4]. Furthermore, genetic factors have also been implicated in gastric cancer pathogenesis. These include mutations in certain genes, polymorphisms in interleukin family genes, or other deletions and insertions $[5,6]$. Other parameters that have been shown to increase the risk of gastric cancer are tobacco, alcohol, obesity, and chemicals. Smokers have 1.53-fold increased risk of gastric cancer, compared to nonsmokers, while alcohol increases the risk approximately by $40 \%$ [7, 8]. Increased BMI is highly associated with increasing risk of developing gastric cancer, since obesity contributes to inflammations through different factors (TNF- $\alpha$ and IL-6) [9]. Gastric cancer is a disease with higher heterogeneity both in molecular aspectand in phenotype. The treatment protocols differ depending on the stage of the disease and include endoscopic resection up to chemotherapy for advanced stages [10]. Recent studies demonstrated that the benefit after first-line therapy is limited, while second-line therapy is adequate for specific patients. Furthermore, the third- or later-line treatment is a potential solution mainly for metastatic gastric cancer; however, there are also a lot of limitations [11]. It is therefore clear that screening is of primary importance in gastric cancer. Most common screening methods involve contrast radiography and endoscopy and noninvasive testing like serum pepsinogel levels, serum trefoil factor 3, or H. pylori serology test [12]. Liquid biopsy, focusing in circulating tumor cells (CTCs), cancer stem cells (CSCs), circulating-free DNA (cfDNA), and extracellular vesicles, assists not only in early detection but also in prediction of treatment response and prognosis of the disease. In the present study, we examined 2 gastric cancer cases, performing a comprehensive analysis, including detection of mutations, detection of fusions using next-generation sequencing (NGS), chromosomal abnormalities via array comparative genomic hybridization (aCGH), and gene expression analysis through microarrays. Based on the above, we aim to assess whether this comprehensive analysis could reveal new data, beneficial for prediction or prognosis.

\section{Methods}

\section{Samples}

The first patient (coded as CS-CC-002) was a male, born in 1949, with gastric cancer, stage III. According to ECOG performance criteria [13], this patient is in status 1 (restricted in physically strenuous activity but ambulatory and able to carry out work of a light or

\section{Karger's}


sedentary nature, e.g., light house work and office work). There is no family history, and the patient had partial gastrectomy in the October of 2020 , followed by adjuvant chemotherapy. In addition, the patient is not a smoker, does not consume fatty foods, does not drink alcohol, and there is no history of inflammations, autoimmune colitis, and H. pylori infection.

The second patient (coded as CS-CC-004) was a male, born in 1963, with gastric cancer, stage IV. The ECOG status is 0 (fully active, able to carry on all pre-disease performance without restriction). There is no family history, and the patient receives therapy for the first time. He is a nonsmoker, nonalcohol consumer, and has obesity GII. Finally, there is no history of inflammations, autoimmune colitis, and $H$. pylori infection.

\section{Blood Sample Preparation}

Thirty milliliters of whole blood was incubated with $20 \mathrm{~mL}$ of ammonium chloride solution (154 mM NH${ }_{4} \mathrm{Cl}$ 31107; Sigma-Aldrich; 10 mM $\mathrm{KHCO}_{3}$ 4854; Merck, Darmstadt, Germany; and 0.1 mM EDTA in deionized water) for erythrocyte lysis. Cells were incubated with the solution for $10 \mathrm{~min}$ under agitation and then centrifuged for $5 \mathrm{~min}$ at 2,000 rpm. Supernatant was discarded, and cell pellet was washed with $20 \mathrm{~mL}$ PBS. After centrifugation at $5 \mathrm{~min}$ at 2,000 rpm, the supernatant was discarded, and cell pellet was incubated with pancytokeratin beads recognizing CK4, CK5, CK6, CK8, CK10, CK13, and CK18 (5c-81714; Gentaur) at $4^{\circ} \mathrm{C}$ for $15 \mathrm{~min}$. Following incubation, the samples were placed in a magnetic rack for panck-positive fraction collection. Pan-ck-positive cells (cancer cells) were washed with $10 \mathrm{~mL}$ PBS for $5 \mathrm{~min}$ at 2,000 rpm. After centrifugation, the supernatant was discarded. The cell pellet was split in two. Half of the cells were used for RNA extraction, and half of the cells were used for DNA extraction.

\section{Next-Generation Sequencing}

Genomic DNA was isolated with the QIAamp DNA Mini Kit (51306; Qiagen, Hilden, Germany) and evaluated with Qubit Fluorometric Quantification and Qubit ${ }^{\mathrm{TM}}$ 1X dsDNA HS Assay Kit (Q33231; Invitrogen, Waltham, MA, USA). Libraries were prepared with TruSight Oncology 500 Kit (ILL.20032627; Illumina, San Diego, CA, USA), evaluated with qPCR (KK4844), and run on an Illumina's NextSeq 550 analyzer. The analysis was performed with Clinical Genomic Workspace, v6.15.1, powered by pieriandx.

\section{Array Comparative Genomic Hybridization}

Genomic DNA was isolated with QIAamp DNA Mini Kit (51306; Qiagen). The aCGH protocol was performed using the Sureprint G3 human CGH 8x60K platform (G4450A; Agilent, Santa Clara, CA, USA) according to the manufacturer's instructions. Analysis was performed with Cytogenomics, v5.2.

\section{Gene Expression Microarray Data}

Total RNA was extracted using an RNeasy Mini Kit (74105; Qiagen). A reference sample (Universal Human Reference RNA, 740000-41; Agilent) was also used in the experiments. Preparation of samples for hybridization followed with Low Input Quick Amp Labeling Kit (5190-2305; Agilent) and hybridization continued with Gene Expression Hybridization Kit (5188-5242; Agilent), into SurePrint G3 Human Gene Expression v3 8x60K Microarray slides (G4851C; Agilent), according to manufacturer's instructions. Sample slides were washed with Gene Expression Wash Buffer Kit (5188-5327; Agilent) and were imaged on an InnoScan 710 (Innopsys) with 5- $\mu$ m resolution. Scanned microarray image data were processed using Array-Pro Analyzer 6.3 Software. The microarray gene expression data were normalized using background subtraction.

\section{Karger's}


Data Preprocessing

The raw data were assessed through the R statistical software and more specifically with the help of the VSN statistical package [14, 15], which implements a method for normalizing microarray data. In brief, it is a model that incorporates a data calibration step, a model for the dependence of the variance on the mean expression, and a variance stabilizing data transformation. The preprocessing procedure also involved background correction and data condensing. More specifically, the probe IDs were transformed into genes by eliminating those with no gene symbols, and areas without any expression were also removed from our study. In cases of multiple probes for the same gene, the mean value was evaluated, producing a single expression per gene. The result yielded 7,487 proteincoding genes.

\section{DEGs Discovery and Classification}

The differential expression patterns were analyzed and compared to the corresponding normal controls. Specifically, the significant $p$ values for the DEGs were calculated by 2 -sample $t$ tests, defining as a level of statistical significance $\alpha=0.05$ and $\log _{2}$ FC ( $\log$ fold change) $\geq 1.5$ by using $p$ value correction with the help of false discovery rate to minimize false positives [16]. Then, the significant DEGs were subjected to hierarchical clustering analysis, giving as a result 127 overexpressed genes.

\section{Results}

\section{Next-Generation Sequencing}

In patient CS-CC-002, a total of 1,062 variants were observed, with the majority of them being substitutions. There were no detected variants with strong clinical significance (tier I), but we observed variants with potential clinical significance (tier II), variants of unknown clinical significance (tier III), and benign or likely benign variants (tier IV). The tumor mutational burden (TMB) score was 0.8 mut/Mb, which is considered a low score, while the microsatellite instability (MSI) status was stable ( $0 \%$ of unstable sites).

For patient CS-CC-004, the total number of variants were 1,098, consisting mainly of substitutions. No tier I variants were detected, and only one of tier II. The TMB score was 2.6 muts/Mb (medium) and stable MSI status ( $0 \%$ of unstable sites). The tier II and tier III variants are presented in Table 1.

\section{Array Comparative Genomic Hybridization}

Patient CS-CC-002 had an amplification in chromosome X, while CS-CC-004 had the same amplification, as well as a gain in the $\mathrm{Y}$ chromosome. The specific region and length are presented in Table 2 .

\section{Gene Expression Microarray Data}

Genes' expression was normalized to reference RNA sample's expression and compared to the expression levels from normal individuals. More than 100 genes have been identified, which were overexpressed only in patients' samples and not in normal cells. These include transcription factors (ZNF family), receptors, metalloproteinases, and genes implicated in signaling transduction pathways.

The Comparative Toxicogenomics Database [17] was used for the genes that were related to gastrointestinal cancer, based on the information in the previously public literature. Subsequently, we performed relative analysis such as a set-based enrichment for collections of genes using correction of $p$ values.

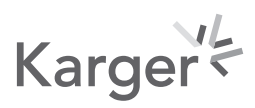


Table 1. Tier II-III variants detected with NGS analysis

\begin{tabular}{|c|c|c|}
\hline Tier & CS-CC-002 & CS-CC-004 \\
\hline II & FGFR4 p.G388R c.1162G>A & - \\
\hline II & SMARCA4 p.Y372H c.1114T>C & - \\
\hline II & TP53 p.P72R c.215C>G & TP53 p.P72R c. 215C >G \\
\hline III & ARID5B p.V651L NM_032199.2 c.1951G>C & $\begin{array}{l}\text { BARD1 p.V507M NM_000465.2 } \\
\text { c.1518_1519delinsCA }\end{array}$ \\
\hline III & BARD1 p.V507M NM_000465.2 c.1518_1519delinsCA & DNAJB1 p.D167E NM_006145.1 c.501C>G \\
\hline III & BARD1 p.I738V NM_000465.2 c.2212A>G & DNMT3B p.A384T NM_006892.3 c.1150G>A \\
\hline III & CARD11 p.A21T NM_032415.4 c.61G>A & EML4 p.E257D NM_019063.3 c.771G>C \\
\hline III & CUX1 p.A654T NM_001913.3 c.1960G>A & FAM46C p.E299K NM_017709.3 c.895G>A \\
\hline III & FANCD2 p.I559V NM_033084.3 c.1675A>G & FANCF p.G225S NM_022725.3 c.673G>A \\
\hline III & FAT1 p.D3317N NM_005245.3 c.9949G> & INHA p.G346R NM_002191.3 c.1036G>C \\
\hline III & GNAS p.R600G NM_080425.2 c.1798C>G & INHA p.P256S NM_002191.3 c.766C>T \\
\hline III & IGF1R p.R1337C NM_000875.3 c.4009C>T & KMT2A p.E502K NM_005933.3 c.1504G>A \\
\hline III & LRP1B p.G935R NM_018557.2 c.2803 G>A & MST1 p.R400C NM_020998.3 c.1198C>T \\
\hline III & MAP3K4 p.K1080R NM_005922.2 c.3239A>G & NOTCH1 p.T596M NM_017617.3 c.1787C>T \\
\hline III & MGA p.T1645I NM_001164273.1 c.4934C>T & PLCG2 p.P522R NM_002661.3 c.1565C>G \\
\hline III & NTRK1 p.H568Y NM_001007792.1 c.1702C>T & PTCH1 p.R682C NM_000264.3 c.2044C>T \\
\hline III & NTRK1 p.G577V NM_001007792.1 c.1730G>T & SOX2 p.Q266E NM_003106.3 c.796C>G \\
\hline III & RANBP2 p.T2602M NM_006267.4 c.7805C>T & \\
\hline III & SDHD p.H50R NM_003002.3 c.149A>G & \\
\hline III & SLX4 p.S1344P NM_032444.2 c.4030T>C & \\
\hline III & SPTA1 p.A970D NM_003126.2 c.2909C>A & \\
\hline III & $\begin{array}{l}\text { ZNF217 p.E914_P915delinsDS NM_006526.2 } \\
\text { c.2742_2743delinsTT }\end{array}$ & \\
\hline III & ZNF703 p.H402Pfs*8 NM_025069.1 c.1205delA & \\
\hline
\end{tabular}

Table 2. Chromosomal abnormalities detected with aCGH

\begin{tabular}{llllll}
\hline Patient & Chromosome & Cytoband & Size, kb & Start-stop position & Type \\
\hline CS-CC-002 & Chr X & p22.2-p11.21 & 44.216 & $13,715,582-57,932,274$ & Amplification \\
& Chr X & q11.1-q28 & 91.707 & $62,062,537-153,771,517$ & Amplification \\
CS-CC-004 & Chr X & p22.31-p11.21 & 48.588 & $9,462,784-58,051,765$ & Amplification \\
& Chr X & q12-q28 & 87.878 & $66,656,771-154,535,178$ & Amplification \\
& Chr X & q21.33-q22.1 & 847 & $97,624,055-98,471,342$ & Amplification \\
& Chr Y & q11.21-q12 & 44.435 & $14,837,758-59,273,048$ & Amplification \\
\hline
\end{tabular}

\section{Discussion}

Gastric cancer has a high mortality rate, which is increased in males and affected by different risk factors, endogenous or exogenous. The treatment protocols differ depending on the characteristics of tumor and include surgery (gastrectomy) where it is operable, usually followed by adjuvant chemo/radio therapy or palliative chemotherapy

\section{Karger'}


in nonoperable cases. The palliative chemotherapy is based on HER2-positive or HER2negative, or other trials with novel agents. After that, second-line chemotherapy is a common treatment practice [18]. Early this year, FDA approved the use of trastuzumab deruxtecan, a second-generation antibody drug conjugate with DNA topoisomerase-I inhibitor, for gastric cancer [19].

Liquid biopsy enabled improvement in treatment decision and/or prognosis of the disease. CTC number and immunophenotype are associated with survival and response. Patients with CTCs $\geq 5$ in $7.5 \mathrm{~mL}$ had shorter disease-free survival and overall survival, compared to cases with lower CTC number [20]. In patients with CTCs expressing CD133, the prognosis was significantly poorer, than in CTCs without expression of this marker, while expression of cytokeratins and CD144 was associated with metastasis [21, 22]. Based on our gene expression assays, the genes that were found to be overexpressed are not limited to those observed in previous studies, but we were able to identify many other overexpressed genes that are involved in various signaling transduction pathways and other cellular processes.

Apart from studying the expression profile of CTCs, detection of variants and fusions could also be beneficial, since they provide useful information for targeted therapies or immunotherapies. The detection of mutations, the calculation of TMB, and evaluation of MSI status have already been evaluated in gastrointestinal cancer [23]. In our analysis, we expanded the number of genes tested and included RNA genes, in order to incorporate any fusions associated with gastric carcinoma. The data revealed plenty of variants, however the majority with unknown or no clinical significance. Furthermore, no fusions were detected as the one of DUS4L-BCAP29, previously reported by Kim et al. [24]. As far as the TMB and MSI status, there were no unstable sites observed, while the TMB score was relatively low, decreasing the possibilities of efficient immunotherapy [25]. In addition, among the different variants detected in both cases, only TP53 p.P72R c.215C>G and BARD1 p.V507M NM_000465.2 c.1518_1519delinsCA are common in patients.

The strategy of comprehensive analysis also includes aCGH, for detection of chromosomal abnormalities [26]. According to previous studies, gains at 1p, 5p, 7q, 8q, 11p, 16p, 20p, and $20 q$ and deletions at $1 p, 2 q, 4 q, 5 q, 7 q, 9 p, 14 q$, and 18q are observed [27]. In the cases we tested, it is noteworthy that all the abnormalities were observed in the $\mathrm{X}$ and $\mathrm{Y}$ chromosomes. These alterations were observed in both cases, indicating a potential pattern. Genetic alterations in the $\mathrm{X}$ chromosome have been implicated in cancer formation and progression [28], while reactivation of X-linked tumor suppressor genes has been a treatment challenge for the last decade [29]. The gained regions of X chromosome are similar to Klinefelter syndrome (XXY), but they do not have a complete extra copy of X, which could contribute to cancer [30]. Their phenotype is also noncompatible with Klinefelter syndrome, making things more complicated.

Based on all the above, it is clearly demonstrated that scientists are able to provide a wide range of information needed for diagnosis and treatment response. On the other hand, physicians need to be educated also in the principles of these techniques, so to know what information could earn in each case. This study is another example of how powerful is the combination among different sciences, including but not limited to biology and medicine. Implementation of mathematics could also enhance the power of this comprehensive panel. We strongly are in favor of using all fields of science to extract all the valuable information that could improve patients' survival and quality of life.

This study is focused on a very limited number of cases; however, the patterns revealed by aCGH and the common genes expressed in both of them, as well as the common mutations, could be used for further studies. Despite the low number of patients, they constitute a representative group of patients, sharing characteristics common in the majority of gastric cancer

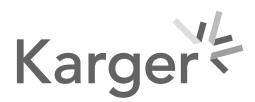


cases. Furthermore, the comprehensive cancer panel used could counteract the small number, by providing a wide variety of information, which could not be extracted just using specific panels, even in a higher number of patients. However, we totally agree that a higher number of samples is required, including long-term information about the progression of the disease and any treatment response. Both the suggested panel, with an appropriate number of cases, could be an ideal proof of this concept.

\section{Conclusions}

Based on the above data, we conclude that one method of screening or diagnosis could not reveal the whole profile of a tumor. The majority of the commonly used methods have advantages and disadvantages. Many of them focus on variants at DNA level, but not all variants affect gene expression, while changes in gene expression levels could be caused not only by mutations but also from many different other parameters, such as activation by other genes or extracellular signals. Therefore, the establishment of a complete cancer comprehensive panel might be more beneficial, since it reveals plenty of information.

\section{Statement of Ethics}

This study is not a clinical trial and does not include intervention in patients. All procedures were conducted according to the standards of Safety, Bioethics, and Validation. The study was reviewed and approved by the Bioethical Committee of the Research Genetic Cancer Centre Group. Each patient has consented in writing for the use of their sample in the present study and for publication. The patients retained the right to withdraw their sample until the date when the sample was received at the laboratory and tested.

\section{Conflict of Interest Statement}

The authors declare that they have no competing interests.

\section{Funding Sources}

No funding.

\section{Author Contributions}

V.P. supervised the study. P.A. carried out the molecular biology assays and drafted the manuscript. G.B. performed analysis of data and drafted the manuscript. E.H. performed cell culturing assays. I.P. supervised the assays and the manuscript.

\section{Data Availability Statement}

The datasets used and/or analyzed during the current study are available from the corresponding author on reasonable request.

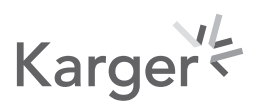


Pisanidou et al.: Comprehensive Analysis in Gastric Carcinoma

\section{References}

1 Rawla P, Barsouk A. Epidemiology of gastric cancer: global trends, risk factors and prevention. Prz Gastroenterol. 2019;14(1):26-38.

2 Bray F, Ferlay J, Soerjomataram I, Siegel RL, Torre LA, Jemal A. Global cancer statistics 2018: GLOBOCAN estimates of incidence and mortality worldwide for 36 cancers in 185 countries. CA Cancer J Clin. 2018 Nov;68(6): 394-424.

3 Matsuda T, Saika K. The 5-year relative survival rate of stomach cancer in the USA, Europe and Japan. Jpn J Clin Oncol. 2013 Nov;43(11):1157-8.

4 Mukaisho K, Nakayama T, Hagiwara T, Hattori T, Sugihara H. Two distinct etiologies of gastric cardia adenocarcinoma: interactions among pH, Helicobacter pylori, and bile acids. Front Microbiol. 2015;6:412.

5 Li J, Woods SL, Healey S, Beesley J, Chen X, Lee JS, et al. Point mutations in Exon 1B of APC reveal gastric adenocarcinoma and proximal polyposis of the stomach as a familial adenomatous polyposis variant. Am J Hum Genet. 2016 May 5;98(5):830-42.

6 Boland CR, Yurgelun MB. Historical perspective on familial gastric cancer. Cell Mol Gastroenterol Hepatol. 2017 Mar;3(2):192-200.

7 Ladeiras-Lopes R, Pereira AK, Nogueira A, Pinheiro-Torres T, Pinto I, Santos-Pereira R, et al. Smoking and gastric cancer: systematic review and meta-analysis of cohort studies. Cancer Causes Control. 2008 Sep;19(7): 689-701.

8 Tramacere I, Negri E, Pelucchi C, Bagnardi V, Rota M, Scotti L, et al. A meta-analysis on alcohol drinking and gastric cancer risk. Ann Oncol. 2012 Jan;23(1):28-36.

9 Tsugane S, Sasazuki S. Diet and the risk of gastric cancer: review of epidemiological evidence. Gastric Cancer. 2007;10(2):75-83.

10 Smyth EC, Nilsson M, Grabsch HI, van Grieken NC, Lordick F. Gastric cancer. Lancet. 2020 Aug 29;396(10251): 635-48.

11 Rizzo A, Mollica V, Ricci AD, Maggio I, Massucci M, Rojas Limpe FL, et al. Third- and later-line treatment in advanced or metastatic gastric cancer: a systematic review and meta-analysis. Future Oncol. 2020 Jan;16(2):4409-18.

12 Kim GH, Liang PS, Bang SJ, Hwang JH. Screening and surveillance for gastric cancer in the United States: is it needed? Gastrointest Endosc. 2016 Jul;84(1):18-28.

13 Oken MM, Creech RH, Tormey DC, Horton J, Davis TE, McFadden ET, et al. Toxicity and response criteria of the Eastern Cooperative Oncology Group. Am J Clin Oncol. 1982 Dec;5(6):649-55.

14 Huber W, von Heydebreck A, Sültmann H, Poustka A, Vingron M. Variance stabilization applied to microarray data calibration and to the quantification of differential expression. Bioinformatics. 2002;18 Suppl 1:S96-104.

15 Huber W, von Heydebreck A, Sueltmann H, Poustka A, Vingron M. Parameter estimation for the calibration and variance stabilization of microarray data. Stat Appl Genet Mol Biol. 2003;2:Article3.

16 Benjamini Y, Drai D, Elmer G, Kafkafi N, Golani I. Controlling the false discovery rate in behavior genetics research. Behav Brain Res. 2001 Nov 1;125(1-2):279-84.

17 Davis AP, Grondin CJ, Lennon-Hopkins K, Saraceni-Richards C, Sciaky D, King BL, et al. The comparative toxicogenomics database's 10th year anniversary: update 2015. Nucleic Acids Res. 2015 Jan;43(Database issue): D914-20.

18 Smyth EC, Verheij M, Allum W, Cunningham D, Cervantes A, Arnold D. Gastric cancer: ESMO clinical practice guidelines for diagnosis, treatment and follow-up. Ann Oncol. 2016 Sep;27(Suppl 5):v38-49.

19 Ricci AD, Rizzo A, Rojas Llimpe FL, Di Fabio F, De Biase D, Rihawi K. Novel HER2-directed treatments in advanced gastric carcinoma: anotHER paradigm shift? Cancers. 2021 Apr 1;13(7):1664.

20 Zhang Q, Shan F, Li Z, Gao J, Li Y, Shen L, et al. A prospective study on the changes and clinical significance of pre-operative and post-operative circulating tumor cells in resectable gastric cancer. J Transl Med. 2018 Jun 20;16(1):171.

21 Xia P, Song CL, Liu JF, Wang D, Xu XY. Prognostic value of circulating CD133(+) cells in patients with gastric cancer. Cell Prolif. 2015 Jun;48(3):311-7.

22 Szczepanik A, Sierzega M, Drabik G, Pituch-Noworolska A, Kolodziejczyk P, Zembala M. CD44(+) cytokeratinpositive tumor cells in blood and bone marrow are associated with poor prognosis of patients with gastric cancer. Gastric Cancer. 2019 Mar;22(2):264-72.

23 Xiao J, Li W, Huang Y, Huang M, Li S, Zhai X, et al. A next-generation sequencing-based strategy combining microsatellite instability and tumor mutation burden for comprehensive molecular diagnosis of advanced colorectal cancer. BMC Cancer. 2021 Mar 16;21(1):282.

24 Kim HP, Cho GA, Han SW, Shin JY, Jeong EG, Song SH, et al. Novel fusion transcripts in human gastric cancer revealed by transcriptome analysis. Oncogene. 2014 Nov 20;33(47):5434-41.

25 Kim J, Kim B, Kang SY, Heo YJ, Park SH, Kim ST, et al. Tumor mutational burden determined by panel sequencing predicts survival after immunotherapy in patients with advanced gastric cancer. Front Oncol. 2020;10:314.

26 Shao L, Kang SH, Li J, Hixson P, Taylor J, Yatsenko SA, et al. Array comparative genomic hybridization detects chromosomal abnormalities in hematological cancers that are not detected by conventional cytogenetics. J Mol Diagn. 2010 Sep;12(5):670-9.

27 Kang JU, Kang JJ, Kwon KC, Park JW, Jeong TE, Noh SM, et al. Genetic alterations in primary gastric carcinomas correlated with clinicopathological variables by array comparative genomic hybridization. J Korean Med Sci. 2006 Aug;21(4):656-65. 
28 Spatz A, Borg C, Feunteun J. X-chromosome genetics and human cancer. Nat Rev Cancer. 2004 Aug;4(8):617-29.

29 Liu Y, Wang L, Zheng P. X-linked tumor suppressors: perplexing inheritance, a unique therapeutic opportunity. Trends Genet. 2010 Jun;26(6):260-5.

30 Swerdlow AJ, Schoemaker MJ, Higgins CD, Wright AF, Jacobs PA. Cancer incidence and mortality in men with Klinefelter syndrome: a cohort study. J Natl Cancer Inst. 2005 Aug 17;97(16):1204-10. 